\title{
عناصر قيمة العلامة كوكاكولا من وجهة نظر المستهلك في ولاية قسنطينة
}

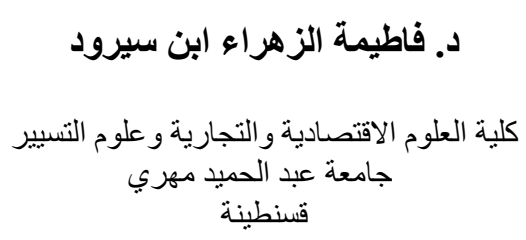

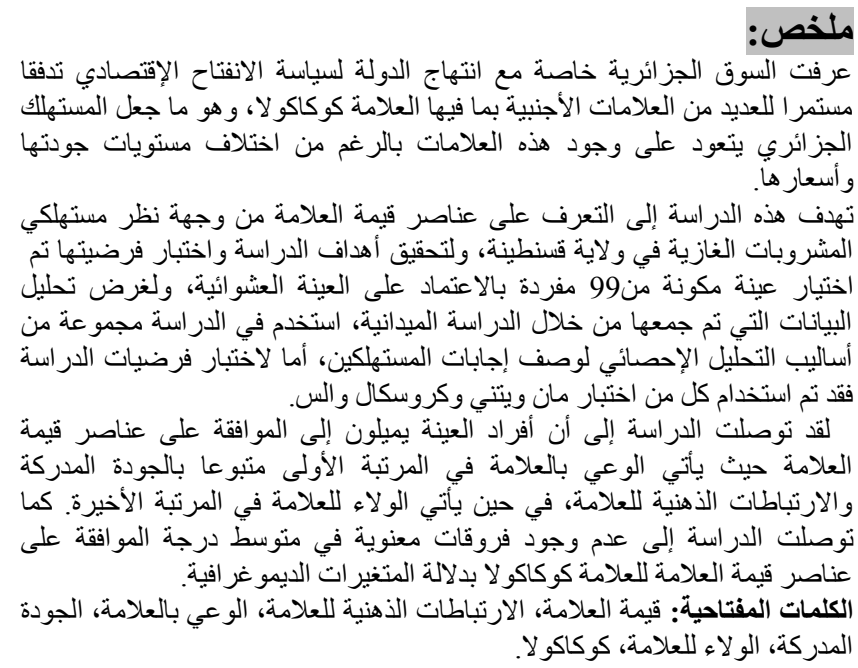

Since the state adopted the economic openness policy, the Algerian market has witnessed a continuous flow of many foreign brands including coca cola, which made the Algerian consumer get used to such brands despite the difference in the levels of its quality and price.

This study aims to identify the elements of the brand equity from the point of view of consumers of soft drinks in Constantine Province, and to achieve the objectives of the study and test the hypothesis. A sample of 99 person has been taken based on a random sample. And in order to analyse the data which has been collected from the field study, a set of statistical analysis have been conducted where arithmetic means, frequencies and percentages were used to describe consumers answers. While Mann-Whitney test and Kruskal-Wallis test were used to evaluate the significant differences in the mean of agreement degree in terms of demographic variables.

The study found that respondents tend to agree on the elements of the brand equity, where brand awareness comes first followed by perceived quality and the mental links of the brand, while the brand loyalty comes lastly. The study also concluded that no significant differences in the mean of agreement degree on Coca cola brand equity elements in terms of demographic variables.

Key words: Brand equity, Brand associations, Brand awareness, Perceived quality, Brand loyalty, Coca cola. 
مع تسارع ونمو العلامات وزيادة المنافسة أصبحت العلامة من أهم وأقوى الأصول غير

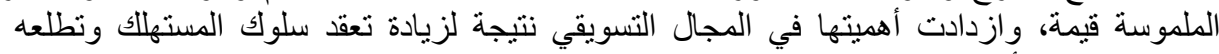

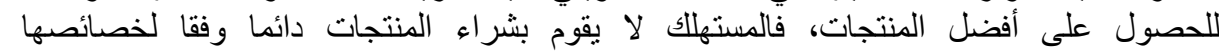

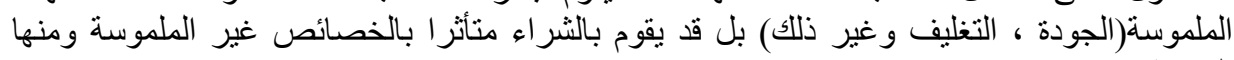

مشكلة الدراسة

تعتبر العلامة بمثابة قوة دافعة في اتجاه تمييز المؤسسات التي تمتلك القدرات الإنتاجية

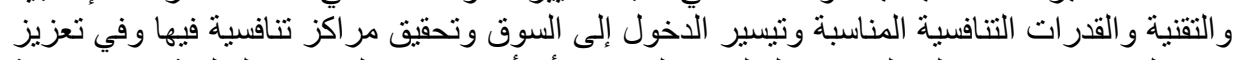

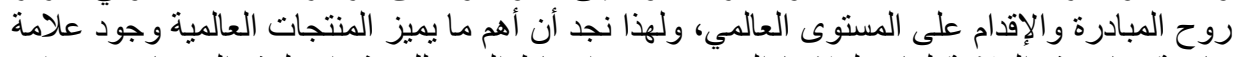

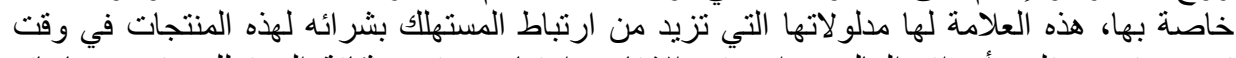

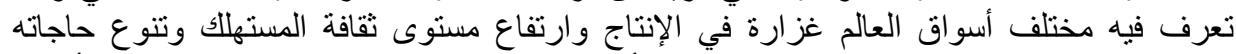

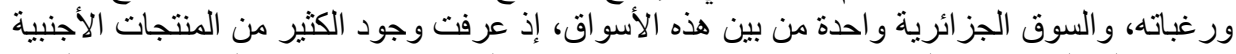

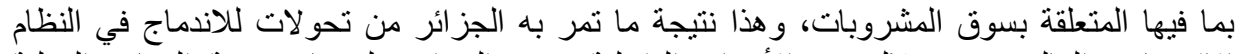

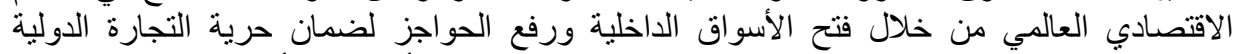

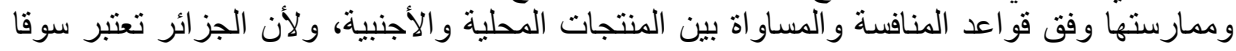

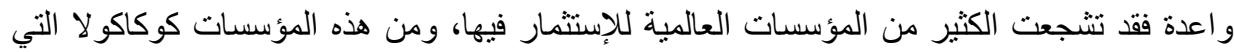

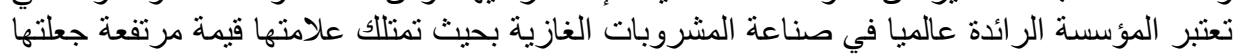

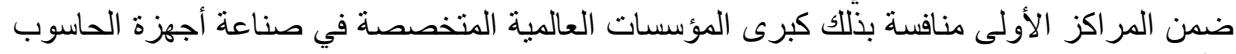
بناء على ما سبق يمكن صباغة السؤ ال الرئيسي الآتي:

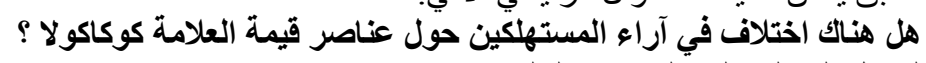
يتفرع هذا السؤ ال إلى السؤ الين الفرعين آراء المين التاليين:

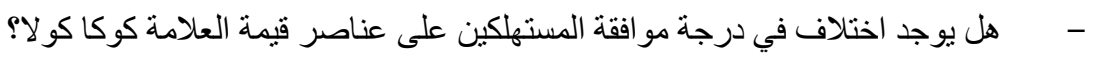

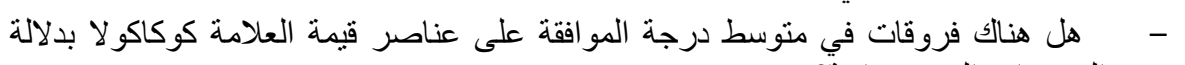

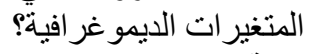
أهداف الدراسة التخغ

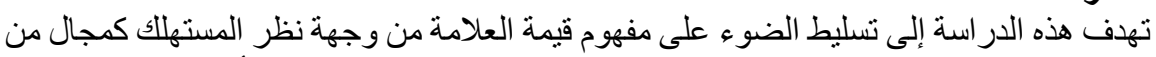

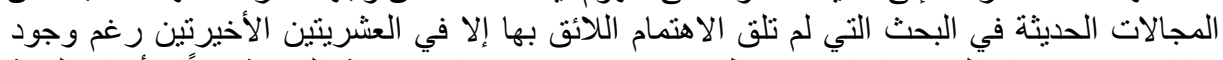

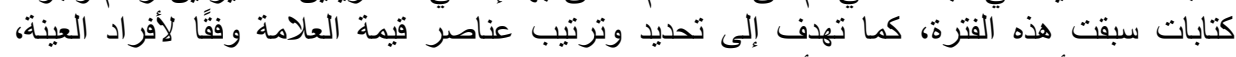

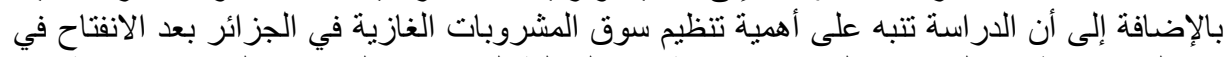

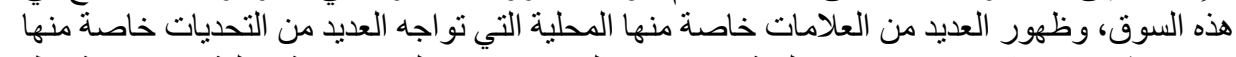
توفير مشروبات ذات مواصفات عالمية من حيث الجودة وترفى لكسب قيمة لئه عالية من وجهة نظر مستخهلكيها. أهمية الدراسة. يمكن إجمال أهمية الدراسة في الأتي:

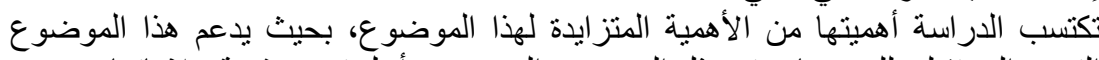

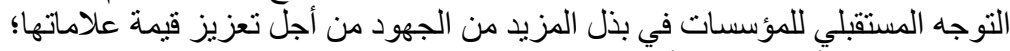

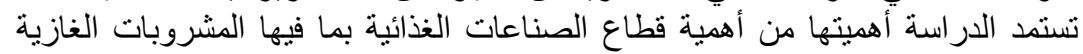

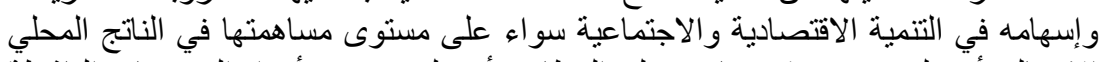

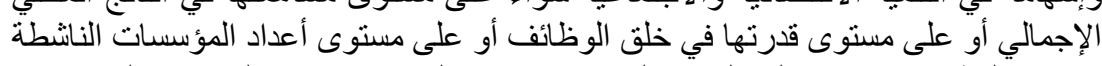

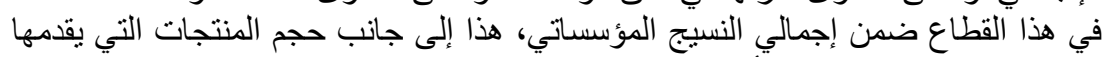

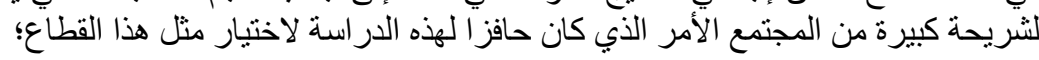


- - الأهمية النظرية و الميدانية التي يمكن أن تسهم نتائج البحث فيها سيما في تعويض النقص في

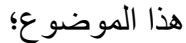

فرضيات الدراسة المبار

تمت صياغة فرضيتين أساسيتين لهذه الدر اسة:

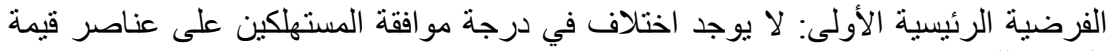

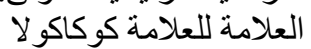

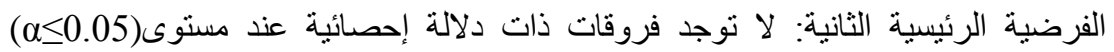

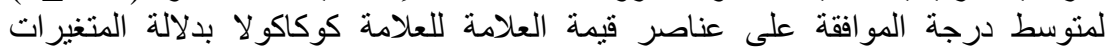

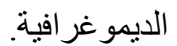

| - الإطار النظري

يعتبر تحقيق تطلعات المستهلكين أو تجاوز ها مهمة لا تخلو من التحديات لا سيما وأن الأذواق

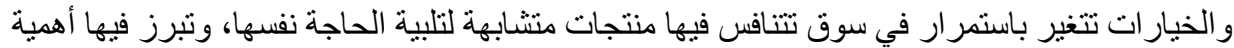
أن تستتد المؤسسة على العلامة باعتبار ها أداة مهمة لتحقيق ميزة منفردة ومتميزة لمنتجات لتهات المؤسسة عن

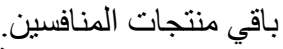

\section{1 1فيهوم وعناصر قيمة العلامة}

يعتبر ظهور العلامة قديما وحديثا في نفس الوقت، إذبا برزت الأنشكال الجنينية للعلامة في عصور

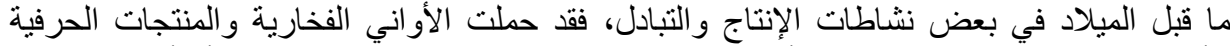

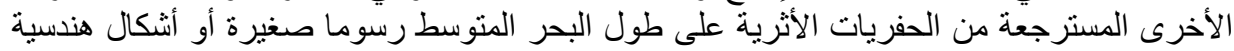

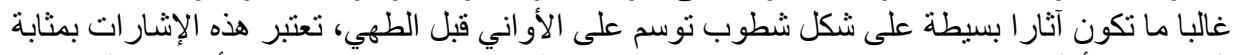

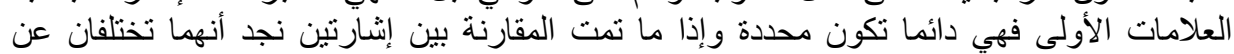

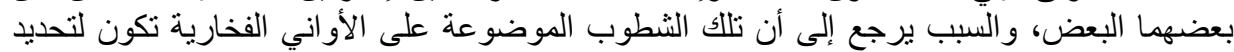

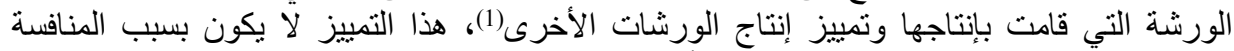

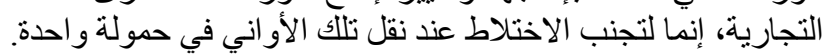

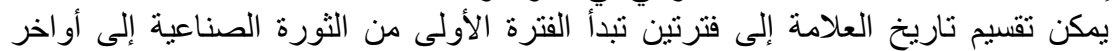

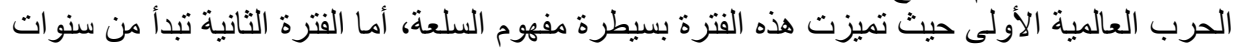

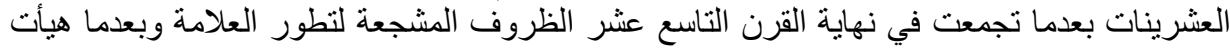

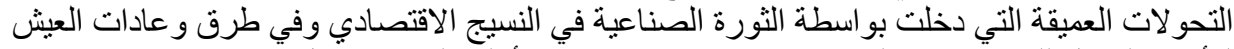

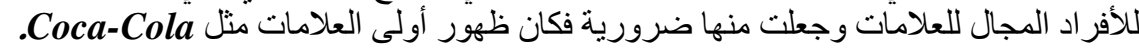

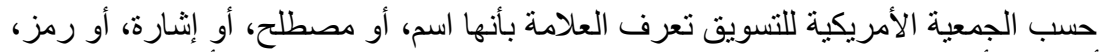

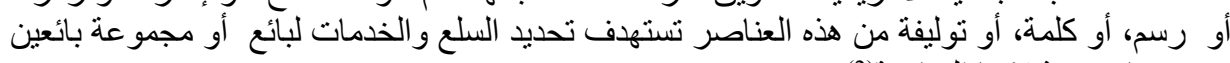

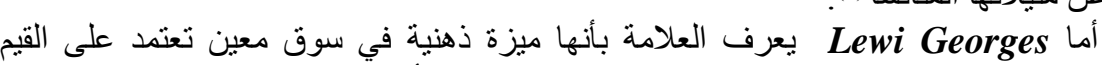

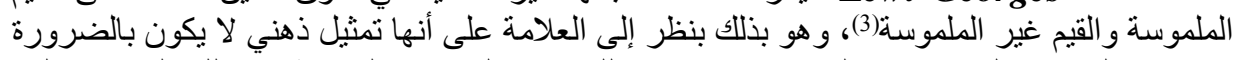

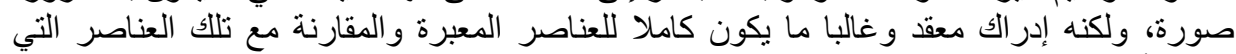

بعثبر مفهوم قيمة العلامة أساس كل الأبحاث الحديثة المرتبطة بتسيير العلامات وتحليل

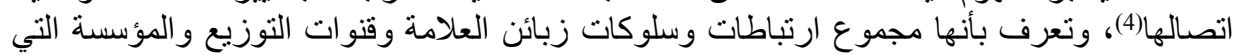

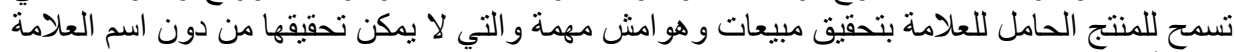

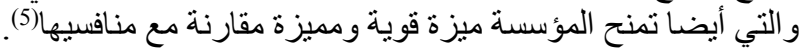
تتضمن قيمة العلامة جانبين هماك(6):

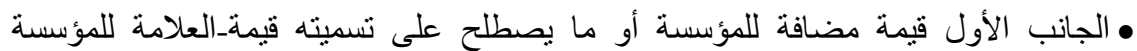
Firm-based equity 
على أنها مصدر للتدفقات النقدية المستقبلية التي تم تحيينها الناتجة عن بيع المنتجات الحاملة لعلامة نسبة

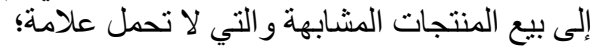

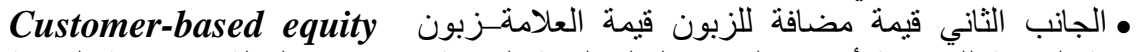

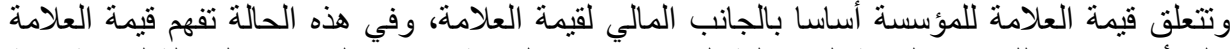

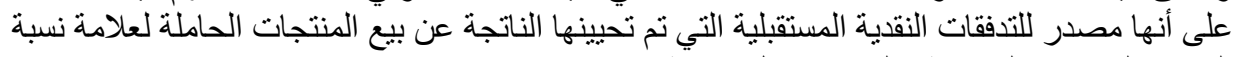
إلى بيع المنتجات المشابهة والتي لاني التحمل علامئ.

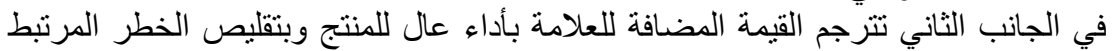

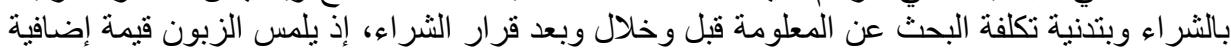

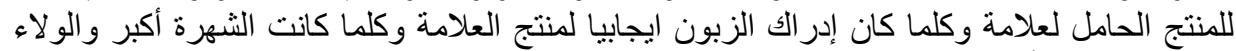

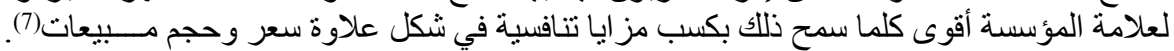

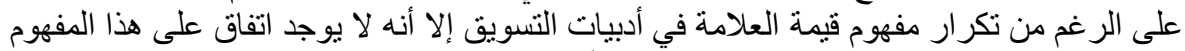

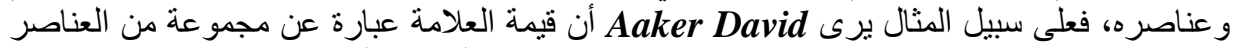

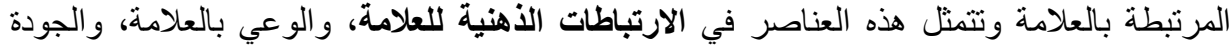

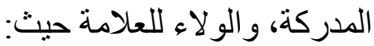

حسب Aaker تعبر الارتباطات الأهنية للعلامة عن أبي شيء مرتبط بذاكرة المستهلك عن الأكن

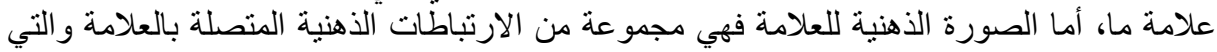
تكون عادة منظمة بطريقة هادفة(8). أما Keller فيرى أن الارتباطات الذهنية للعلامة عبارة عن مجموعة من الارتباطات الذهنية

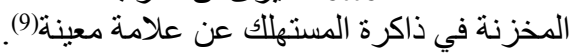

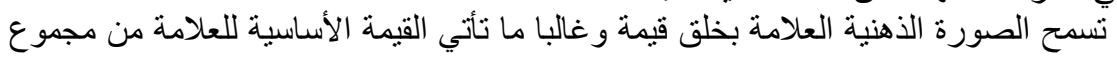

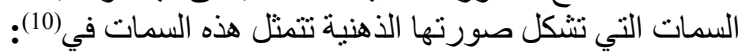

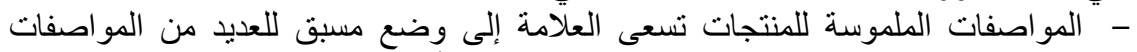

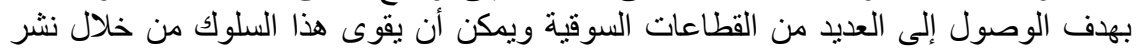
الصورة الذهنية العلامة؛ الصوف الصفيد - الخصائص غير الملموسة للمنتجات إذ تعتبر الخصائص غير الملموسة للمنتجات مو اصفات

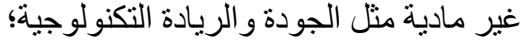

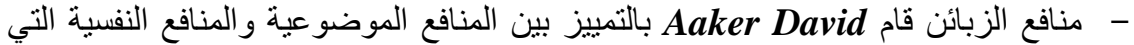

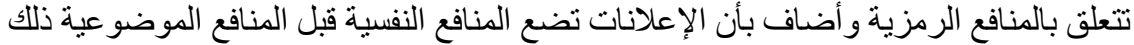

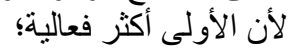

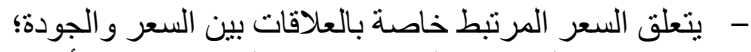

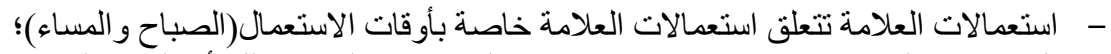

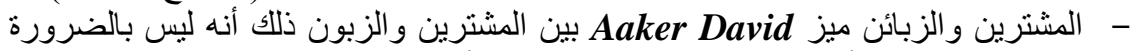

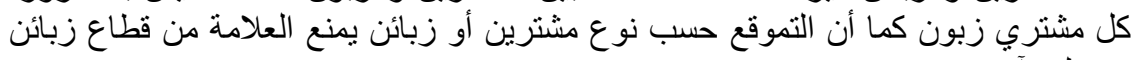

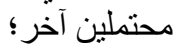

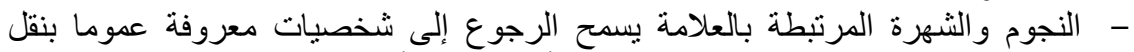

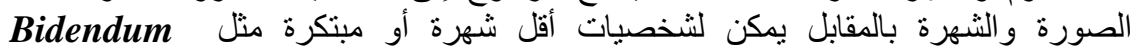
أو Michelin الزبائن؛ - - شخصية وأسلوب حياة العلامة تتعلق بالخصائص الإنسانية المرتبطة بالعلامة.

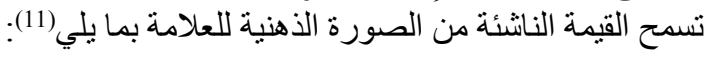




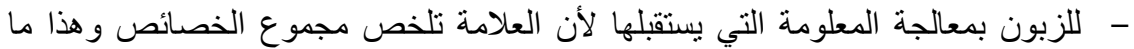

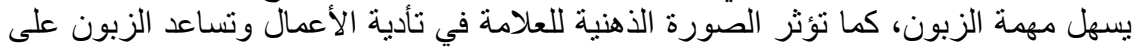

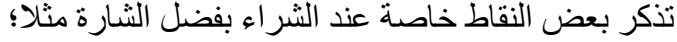

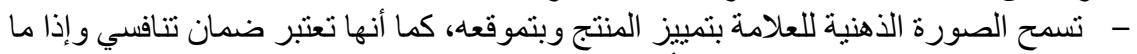

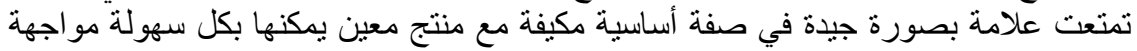

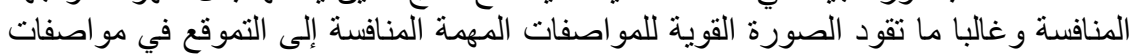

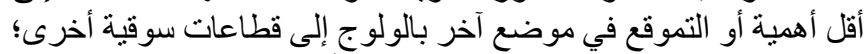

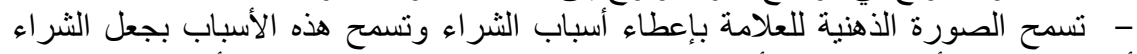

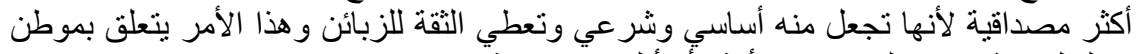

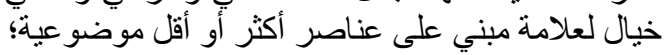

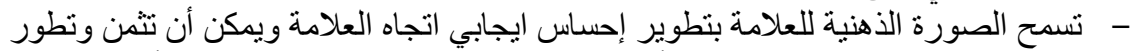

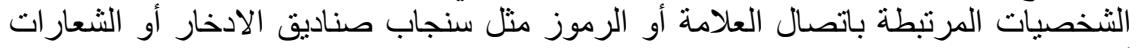
أحاسيس ايجايية تساهم في تغذية الصورة الذية الذهنية للعلامة؛

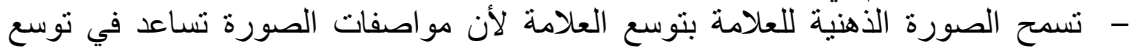

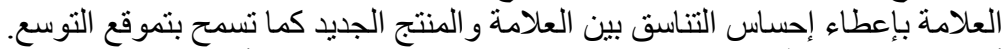

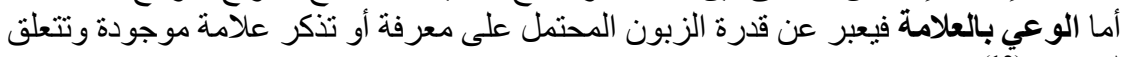
بصنف المنتجات(12).

يرى كل من ( Reller 1993 ( Rossiter \&Percy 1987 ) أن الوعي بالعلامة ينكون من

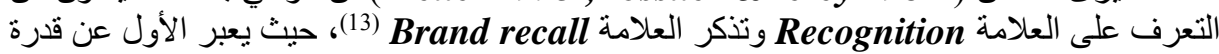

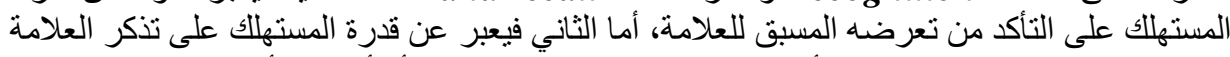

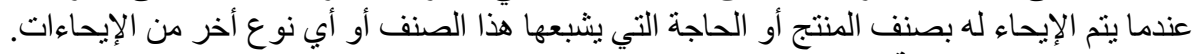

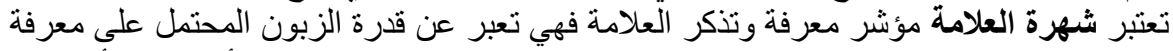

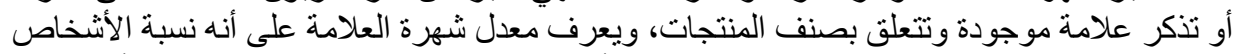

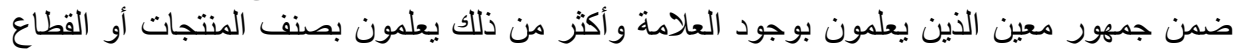
الاقتصادي الذي تنتمي إليه العلامة(14).

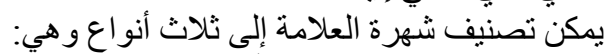

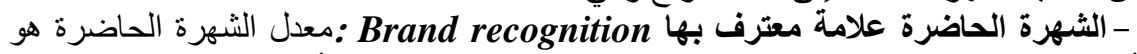

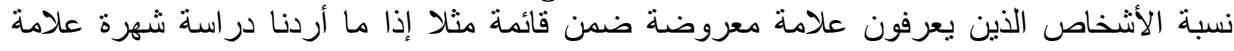

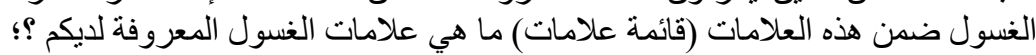

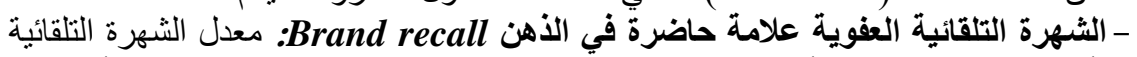
هو نسبة الأثخاص الذين يتمثلون أو يستحضرة علامة حاضرن العلامة عفويا إذن يكون السؤال المطروح أذكر كل علامات الغسول التي تعرفها؛

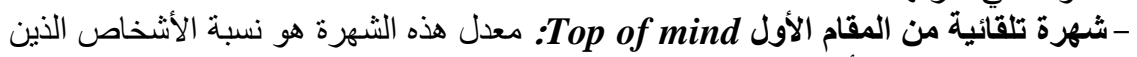

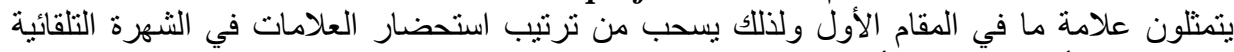

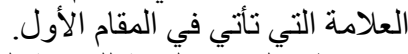

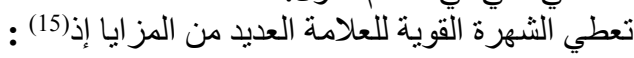

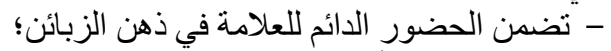
- تمد بإحساس الألفة والاعتياد إذ في حالة عدم توفي الزياني، العلامة المعتاد شراؤها يتجه الزبون إلى

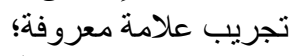

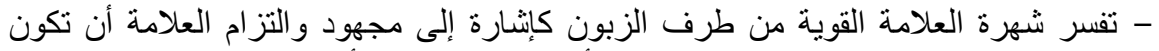

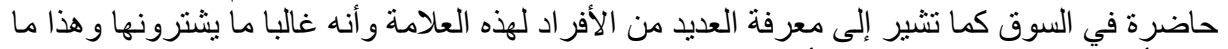
يمكن أن يولد انطباع عن العلامة بأنها ذات جودة؛ 
- بالنسبة للموزعين تثكل الثهرة القوية ميزة لتسعيل المرجعية Le référencement إذ في

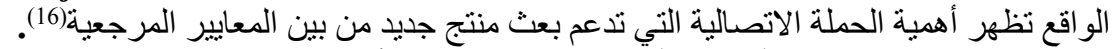

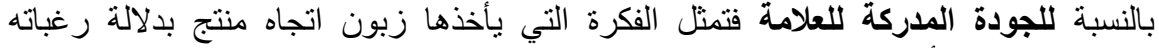

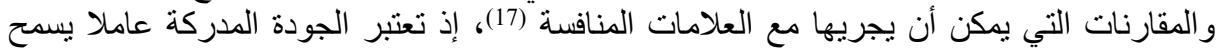

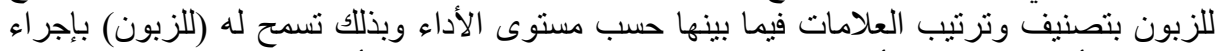

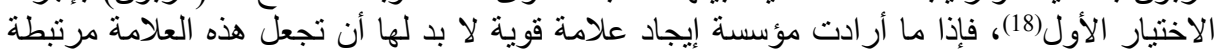

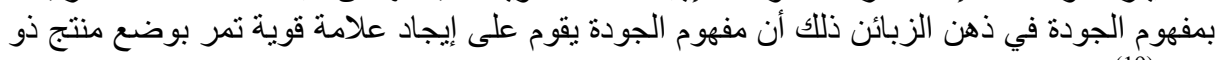
جودة'(19). (19)

$$
\text { - نسمح الجودة المدركة للعلامة البـ (20): }
$$

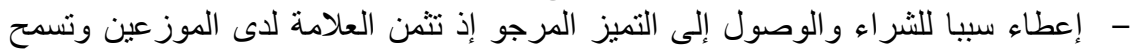
ضمان مهم في حالة توسع العلامة. لألماء.

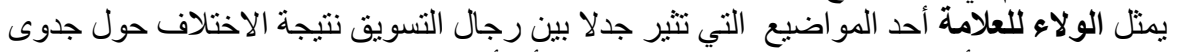

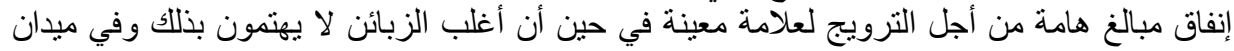

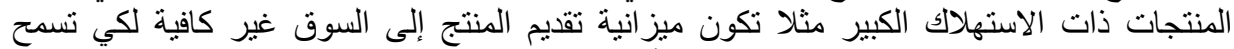

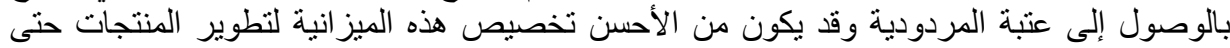

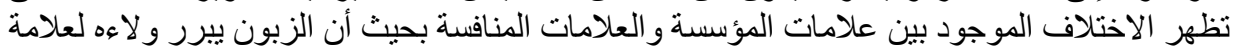
يعرف الولاء للعلامة بأنه قياس ارتباط الزبائن بتلأك العلامة ويعبر عن عدم الميل للتغيير لعلامة

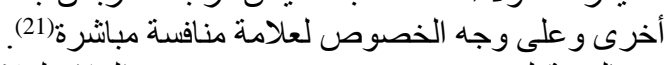

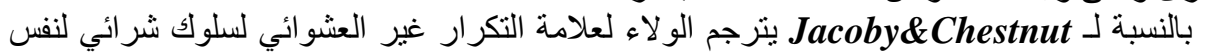

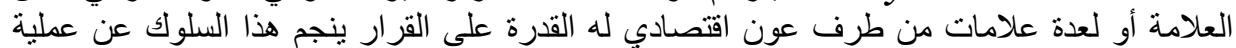
تقبيم تقود إلى اتخاذ القرار (22). يتضح من التعريف السابق أن الو لاء للعلامة يظهر في شكل مجموعة من الثروط الو اجب نو افرها

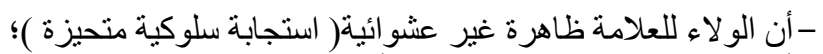

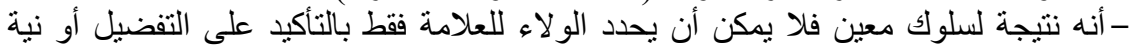
الثراء و إنما يجب أن يتبع ذلك بسلوك شراء اللعلامة ويشترط الاستمرارية في نفس العملية الثر ائية؛ - يمثل نتيجة لعمليات نفسية ويعني ذلك أن الو لاء هو دالة لعمليتي اتخاذ القرار و التقبيم فهو

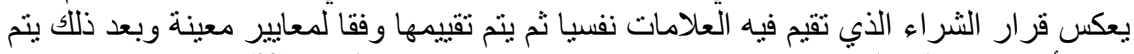

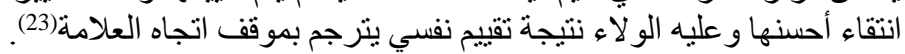

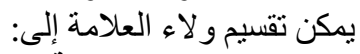

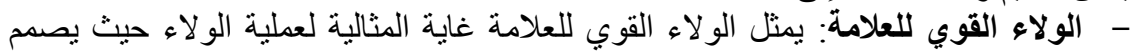

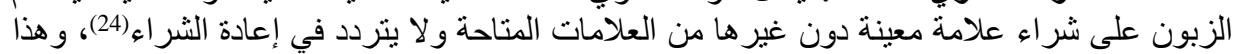

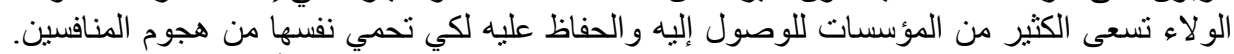

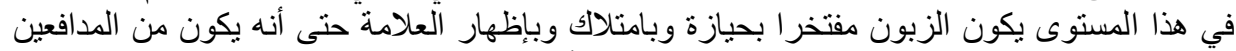

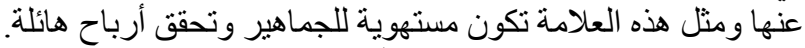

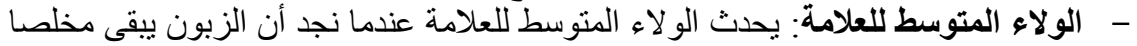

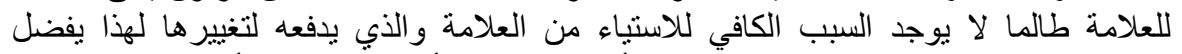

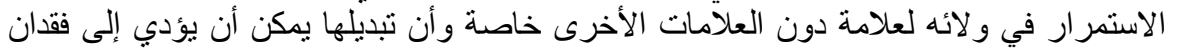


وخسارة الوقت أو أن يكون هنالك خطر أن أداءها أقل، من الأسباب التي تؤدي إلى التحول في و لاء

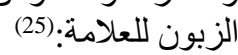

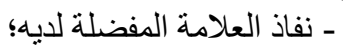

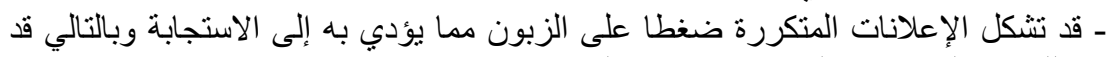
يتغير و لاؤه للعلامة التي تعود الإعنات على استهلاكها في السابق؛

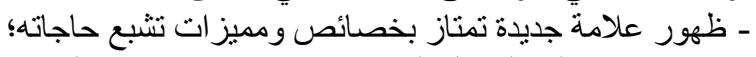

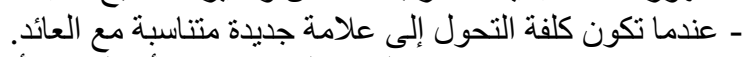

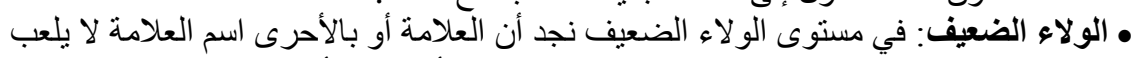

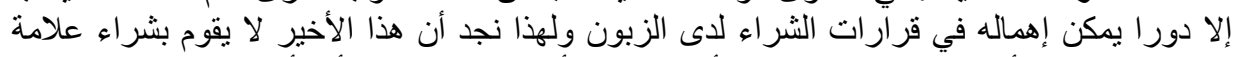

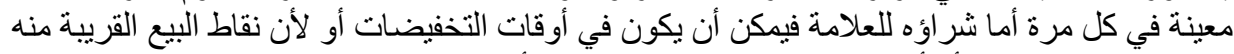

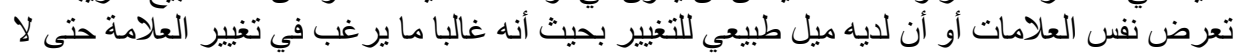
يتولد لديه نوع من الملل اتجاهيا.

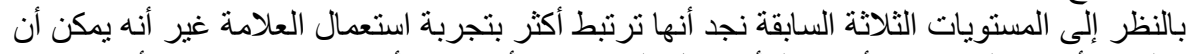

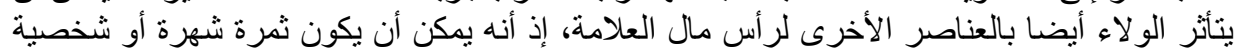

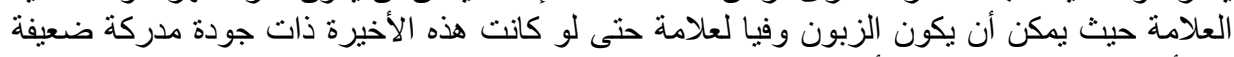

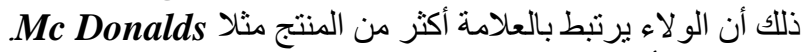

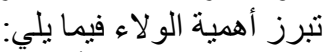

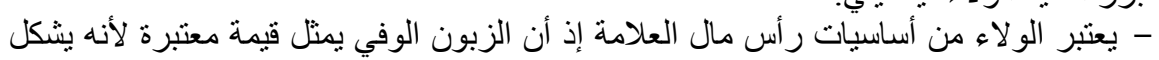

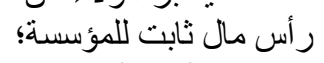

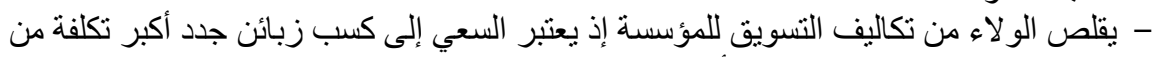

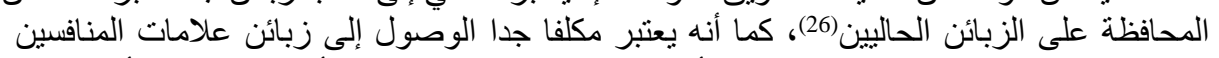

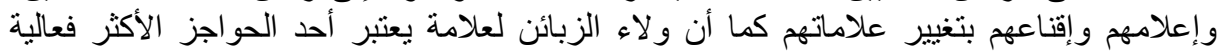

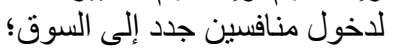

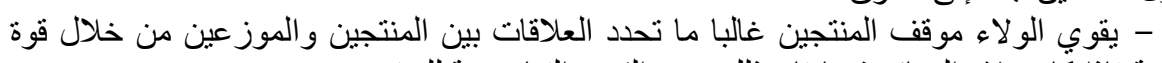

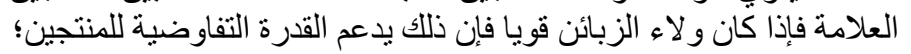

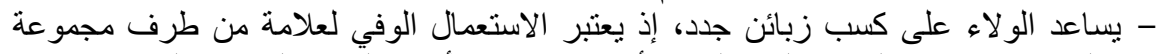

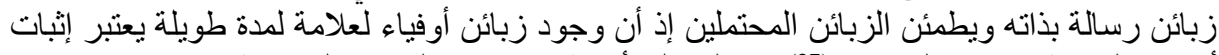

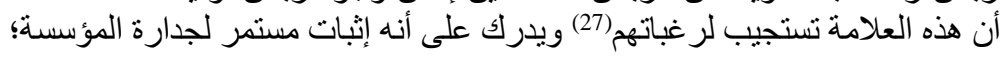

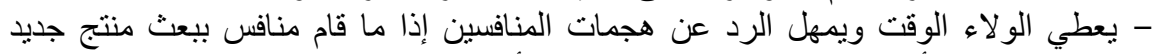

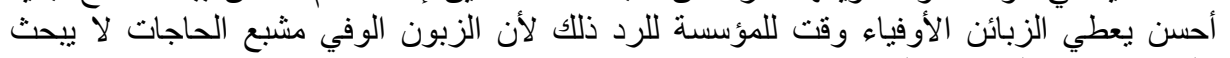

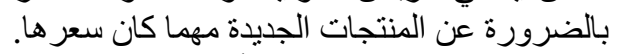

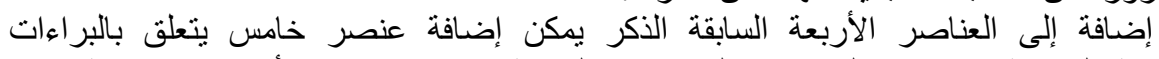

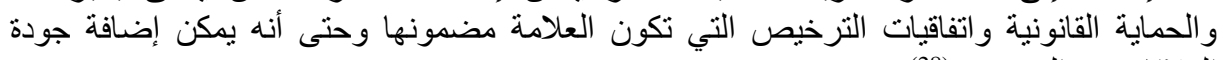
العلاقات مع الموز عين (28).

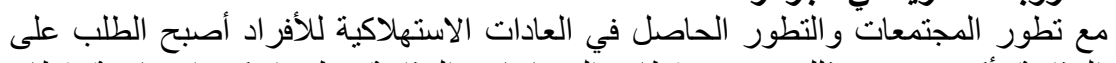
2- قطاع المشروبات الغازية في الجزائر

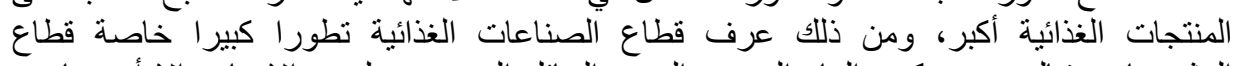

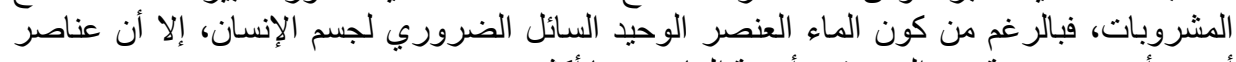

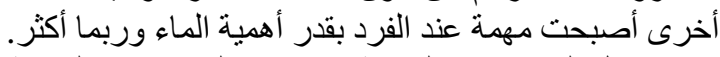

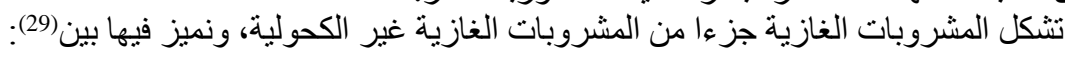

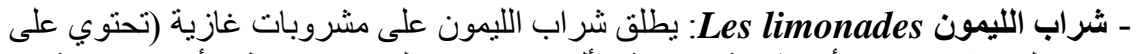

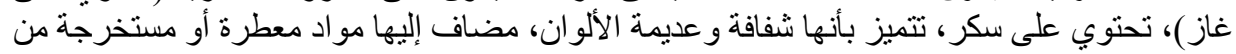


ليمون، وتحنوي كذللك على مواد أخرى*، ويلاحظ أنه من المرخص استخدام السكر والغلوكوز

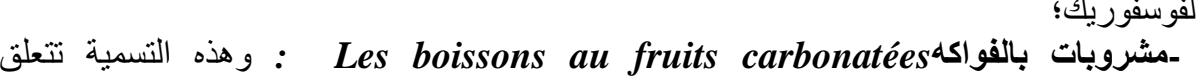

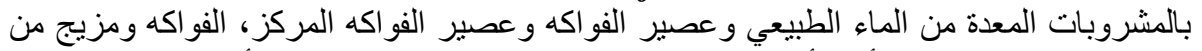

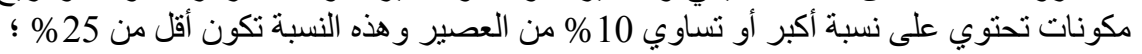

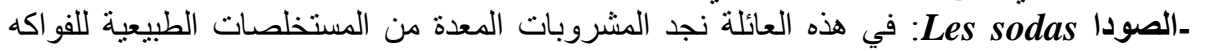

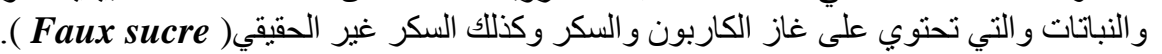

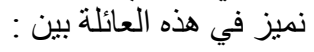

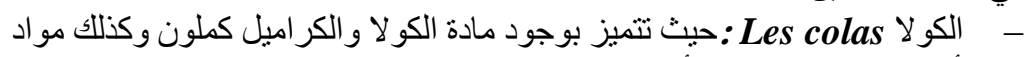

أخرى مثل الكافيين و الأسيد Acide ortho phosphorique

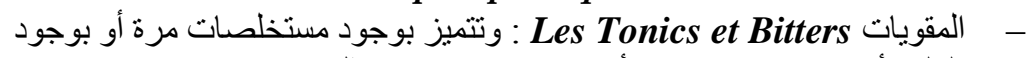

Schweppes : ويمكن أن تكون Limpides وال ونل

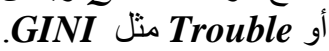

يفرق بين تسمية Tonics أو Bitters بحل فيب مادة Quinine حيث :

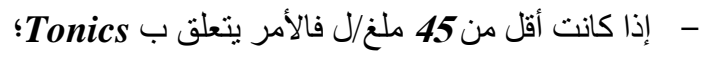

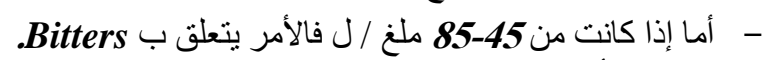

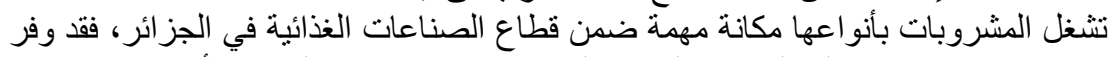

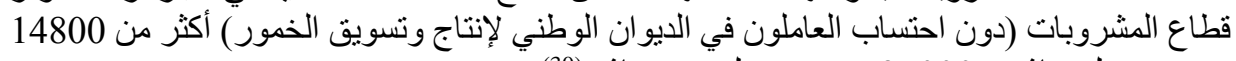

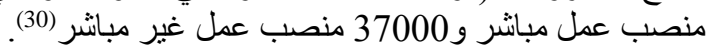

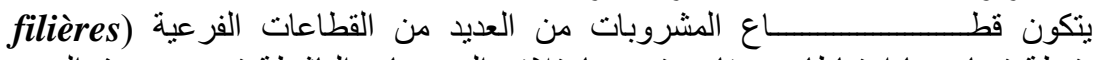

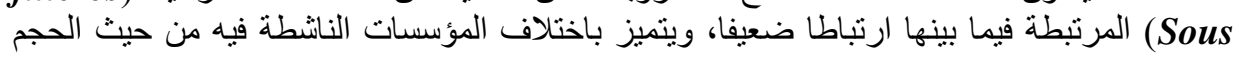

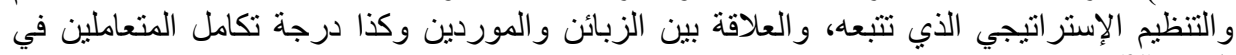

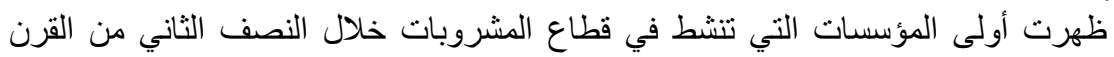

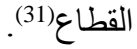

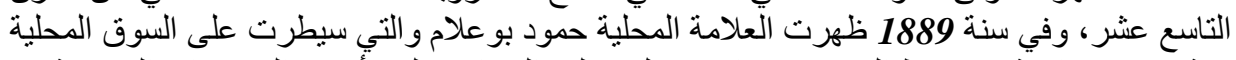

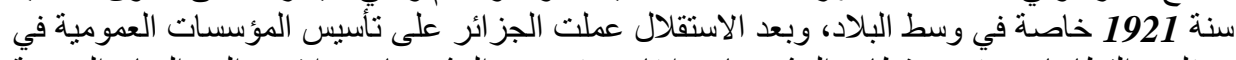

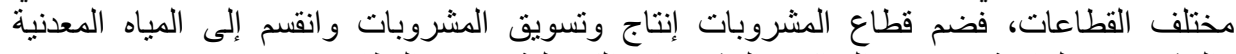

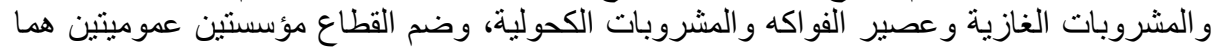
ONCV و الديو ان الوطني لإنتاج وتسويق الخمور

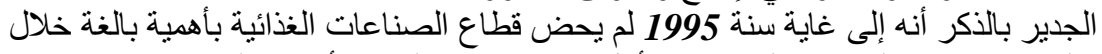

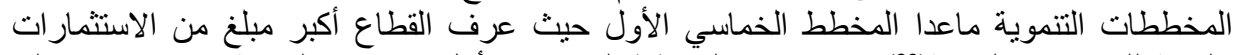

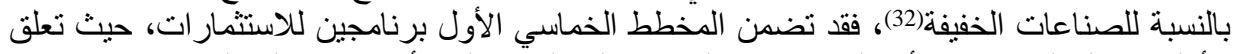

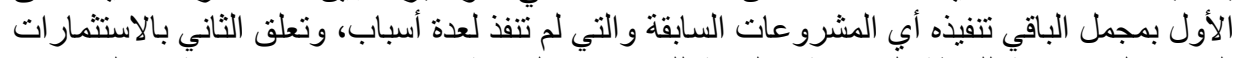

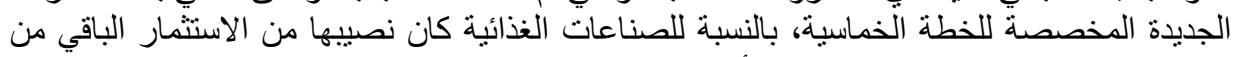

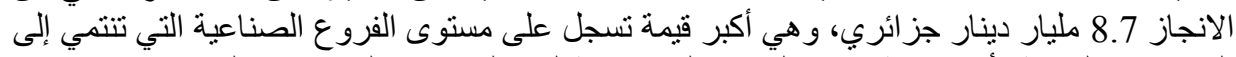

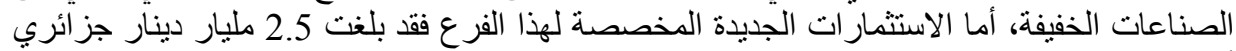
أي بنسبة 7.2 \% من إجمالي الاستثمار ات الكلية في القطاع الصناعي (33).

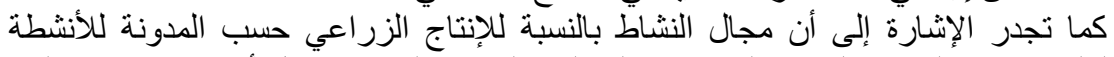

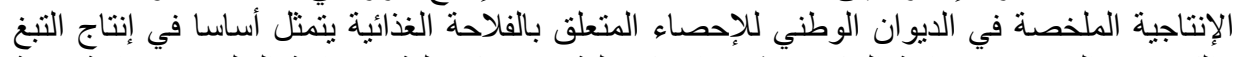

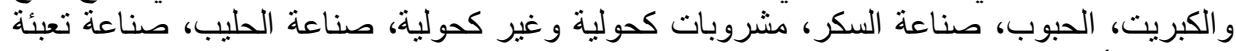
اللحوم و الأسماك و الخضر والفو اكه، صناعات غير معروفة خارجًا كالخمائر ، وصناعة منتجات مغذية 


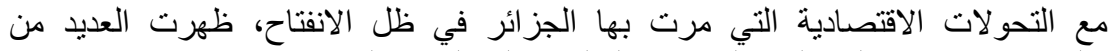

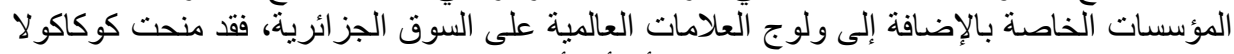

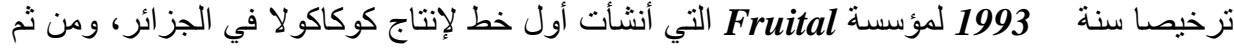
منحت كو كاكو لا ترخيص لـ(•) BGI و التي تعود ملكيتها لمؤسسة كاستل Castel في منطقتي الثرق مما لاشكك فيه أن السوق الجزائرية للمشروبات مقارنة مع مثيلاتها التونسية والمغربية تعتبر

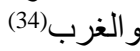

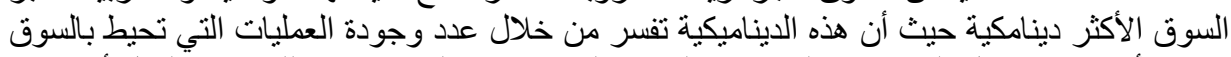

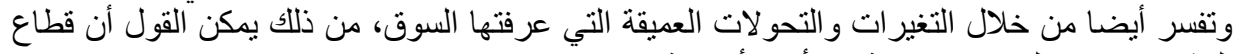

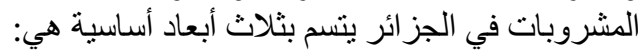

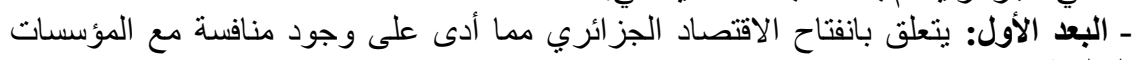

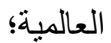
ــ البعد الثاني: يتعلق بالتطور السريع لإنتاج القطاع الخاص وتعدد المتعاملين مثل حمود بو علام و افري وسيدي الكبير و غير هـ؛

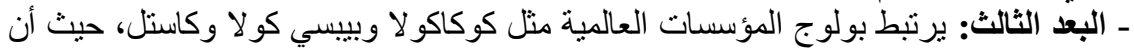

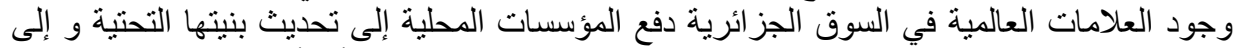

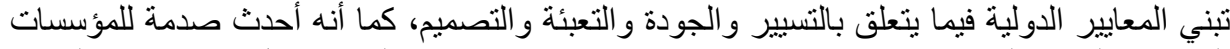

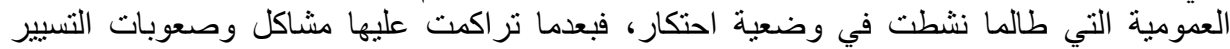

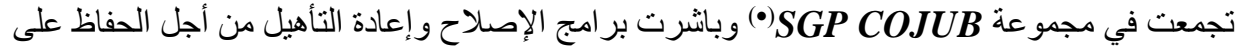

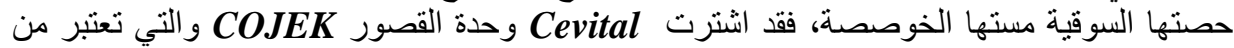

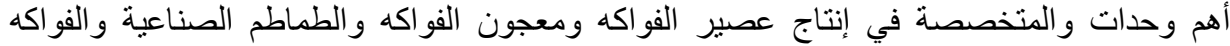

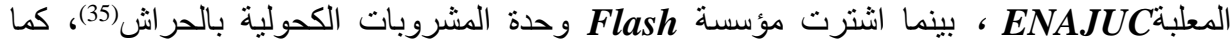
ظهرت جمعية المنتجين الجز ائريين للمشروبات(•)

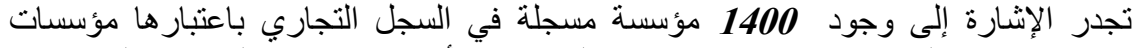

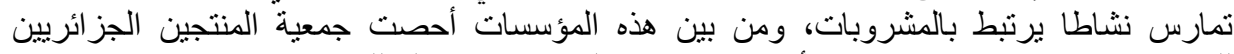

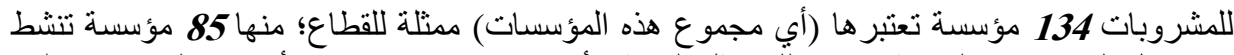

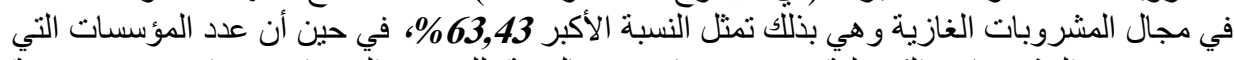

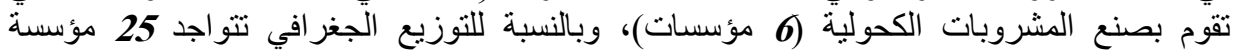

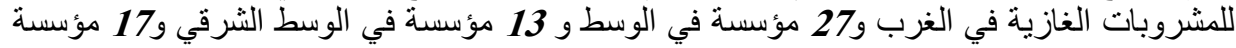

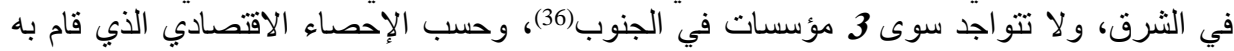

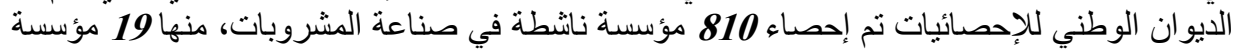

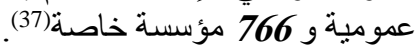

بحسب تقرير EDPME بلغ الاستهلاك السنوي من المشروبات الغازية والمياه المعدنية المعبأة 22 لتر لكل فرد في السنة، وبالنسبة لعصبر الفو اكه والمشروبات بلغ 4.7 لتر لكل لفرد في الكئي

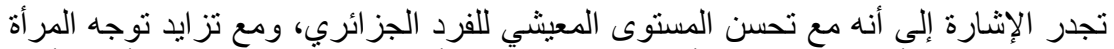

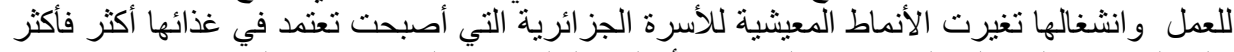

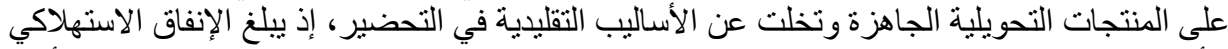

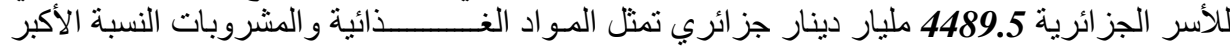

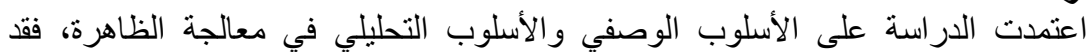
منهجية الاراسة

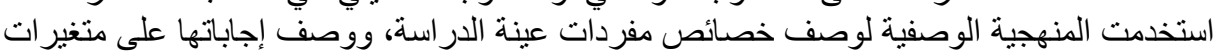




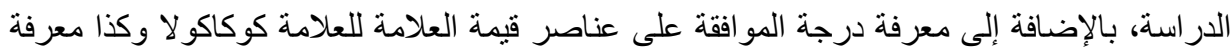
الفروقات بين منوسطات درجة الموافة الفة بدلالة المتغيرات الديموغرافية من خلال التبار الفرضيتة الفيتين

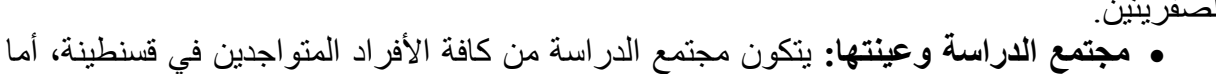

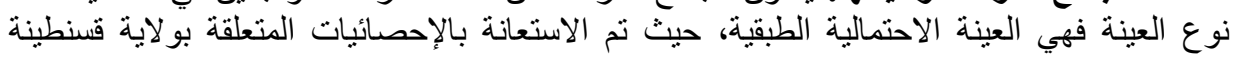

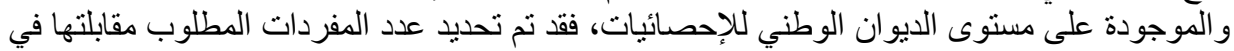

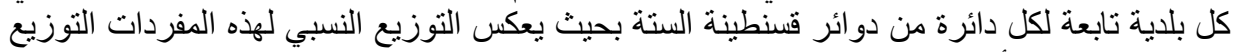

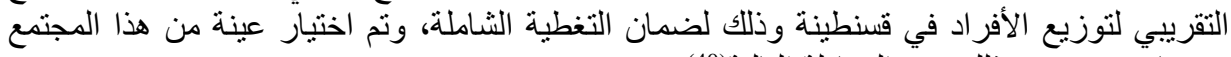
عددها 100 مفردة ذلك وفق المعادلة التالية(40):

\section{$\operatorname{Root}(n)=\operatorname{root}(p)(q) *(1.96 / 0.05)$}

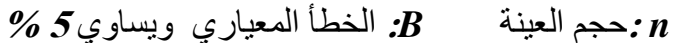

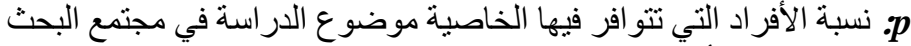

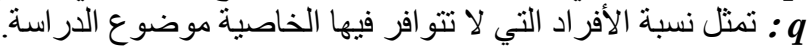

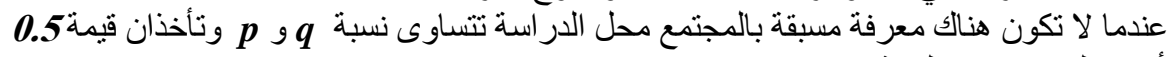
و وهي أحسن لتحديد حجم العينة

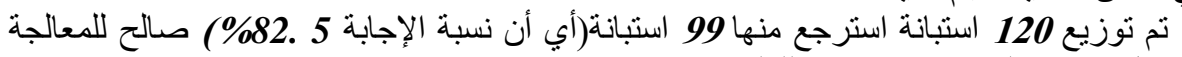

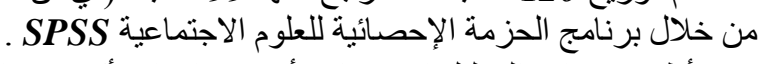

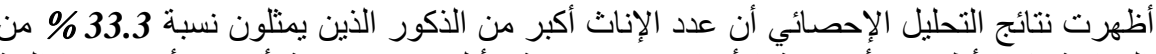

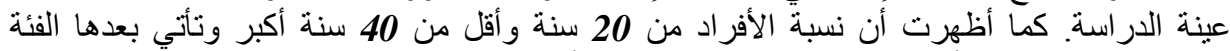

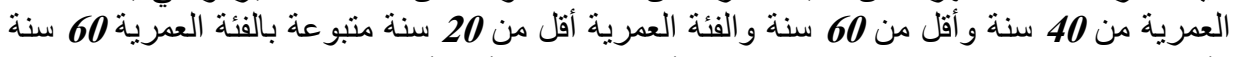

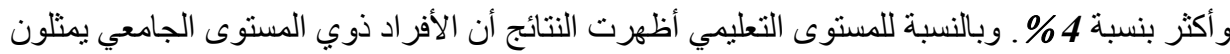

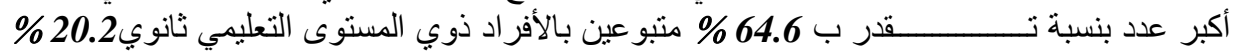

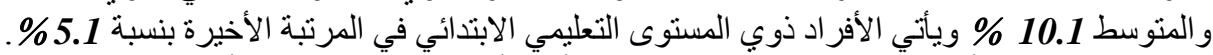

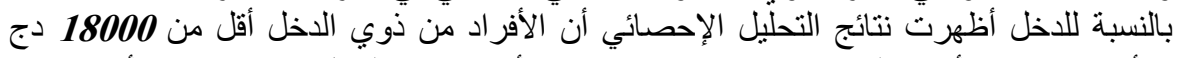

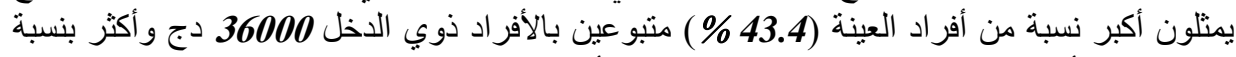

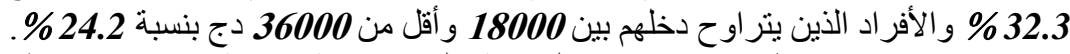

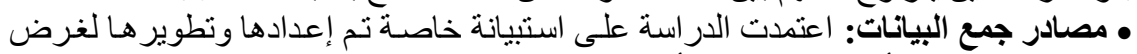

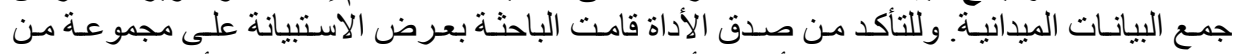

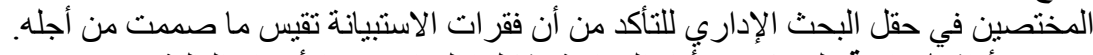

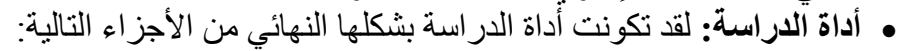

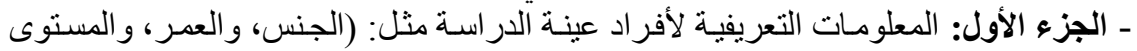

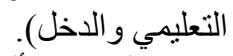

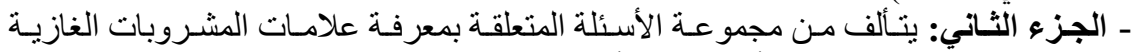

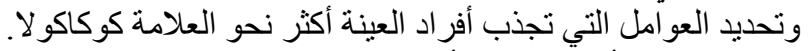

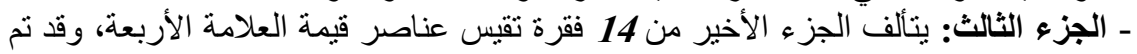

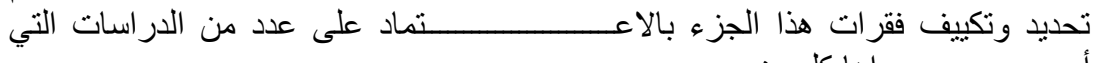

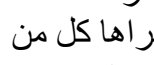

(Yoo Boonghee and Donthu Naveen,2001,Czellar Sandor and Denis JeanEmille, 2001, Jourdan Philippe, 2002,Korchia Michael,2000) 


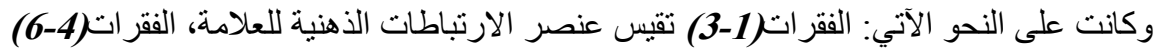

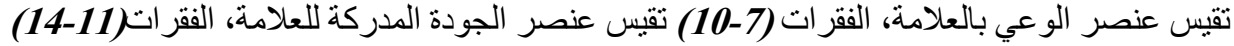

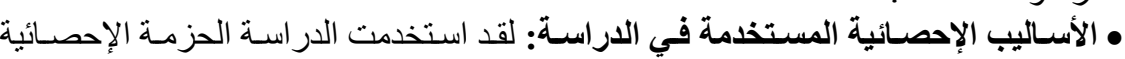
تقيس عنصر الو لاءي للعلامة.

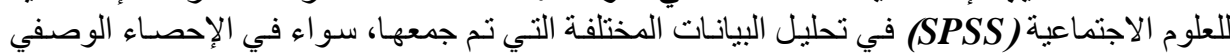

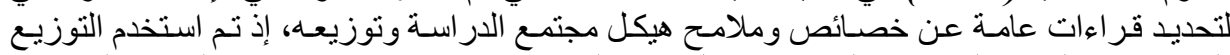

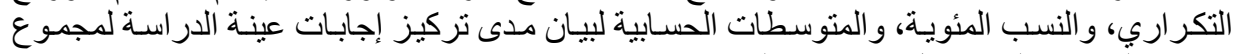

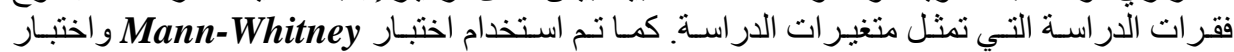
Kruskal-Wallis

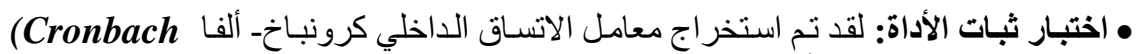
Alpha)

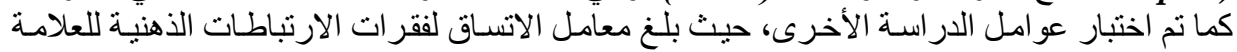

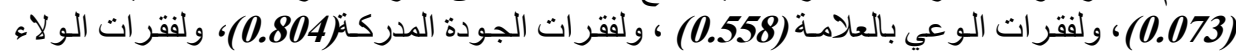

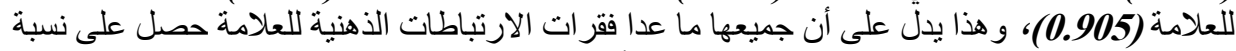

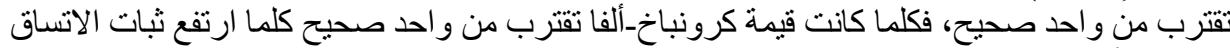

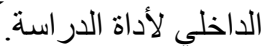

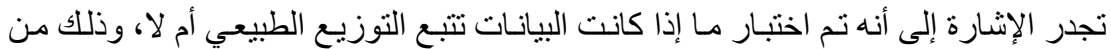

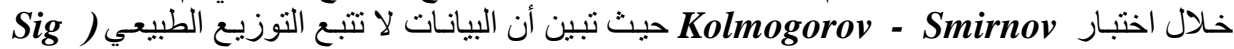
0.05<)، و على ذلك سيتم اللجوء إلى الاختبار ات اللامعلمية لاختبار الفرضية الرئيسية الثانية.

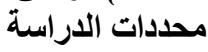
ـ اقتصار عينة الدراسة على 99 مفردة وذلك لرفض بعض الإل الأفراد المشاركة في ملأ الإستبانة

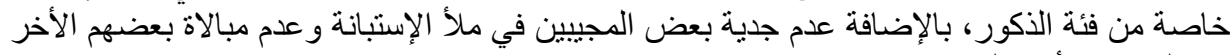

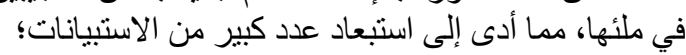

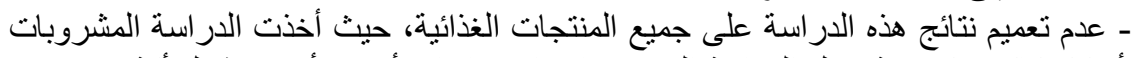

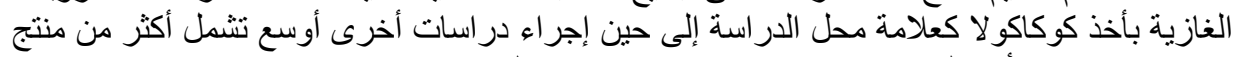

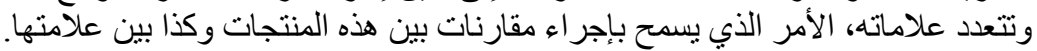
نتائج اختبار فرضيات الارأسة الأبة

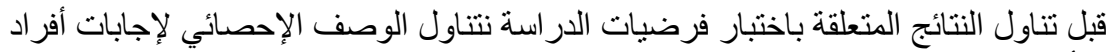

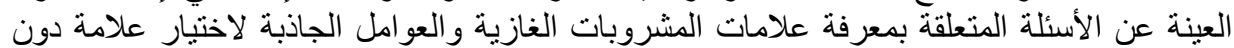
سو اها.

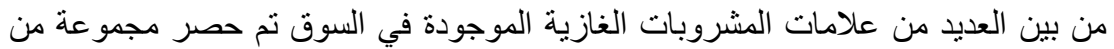

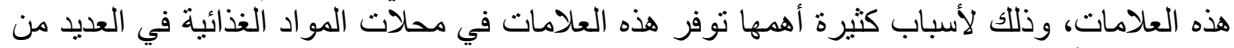

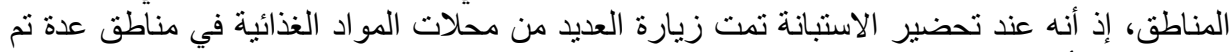

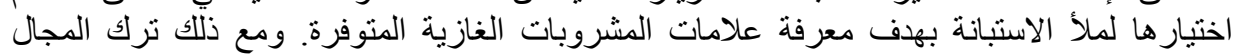

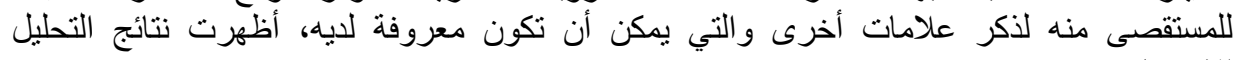
بالنسبة للثهرة التلقائية من المقام الأول أن علامة المشروبات الغازية كوكاكولا تحنل الإحصائي

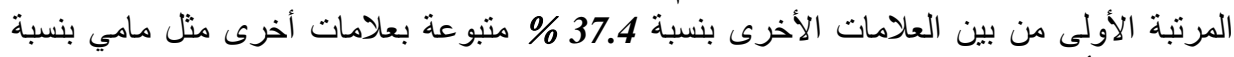

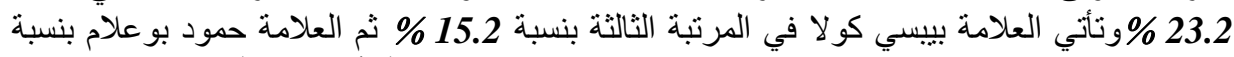

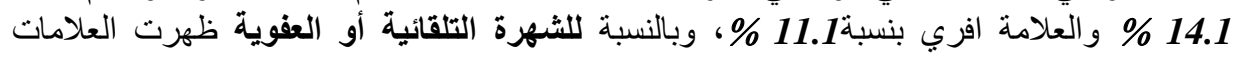

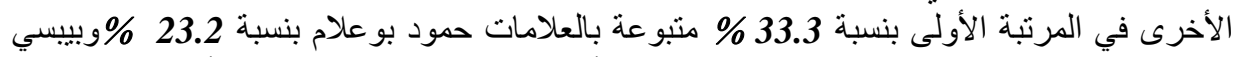
كو لا بنسبة 19.2 \% وكوكاكو لا بنسبة 15.2 \% وتأتي العلامة افري في المرتبة الأخيرة بنسبة 8.1 ولات 455 


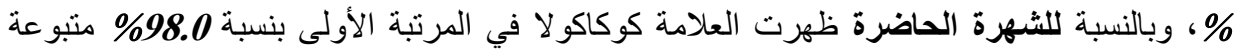

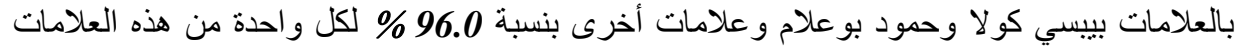

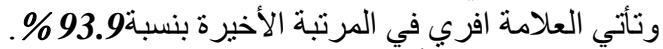

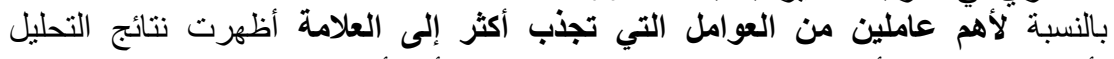

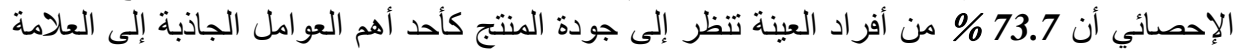

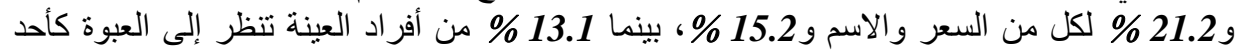

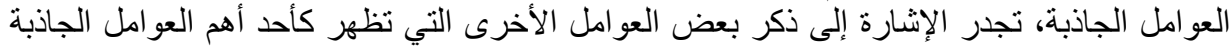

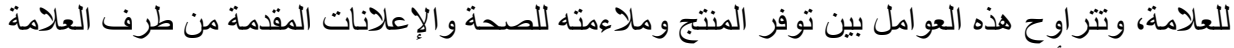

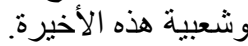

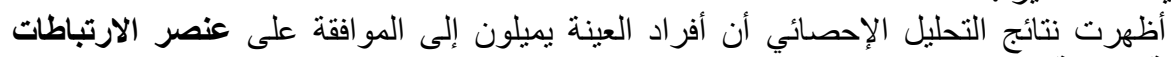

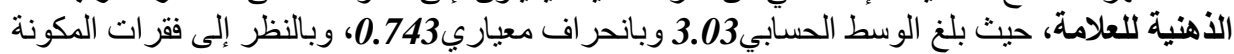

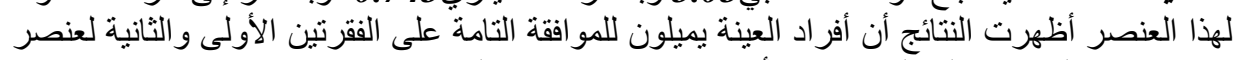

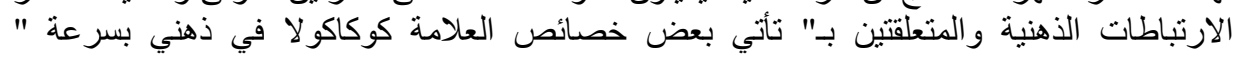

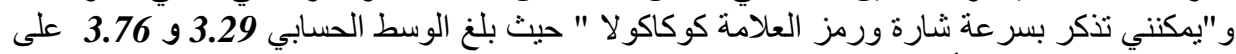

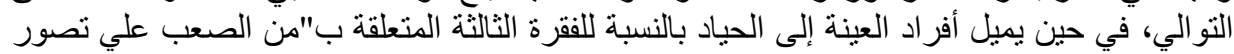

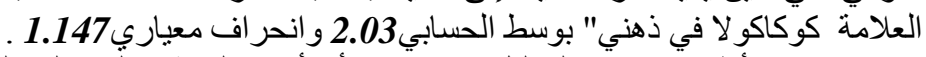

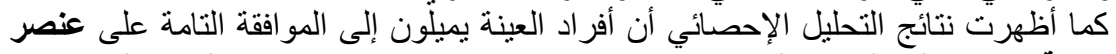
الوعي بالعلامة، حيث بلغ الوسط الحسابي

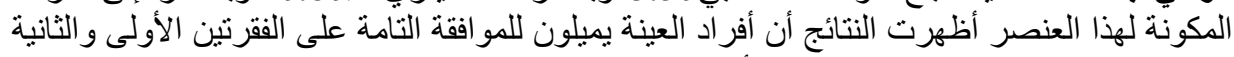

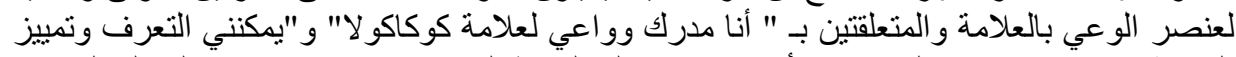

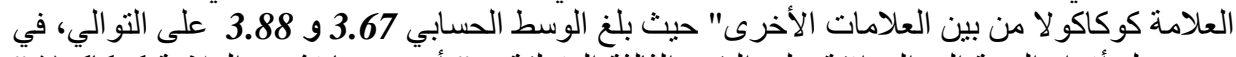

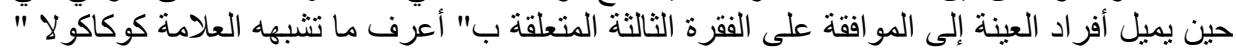

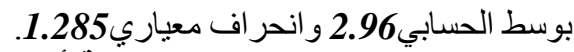

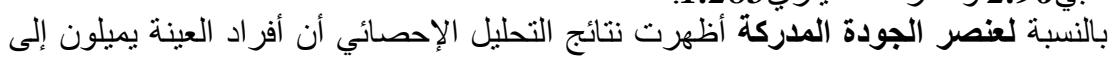

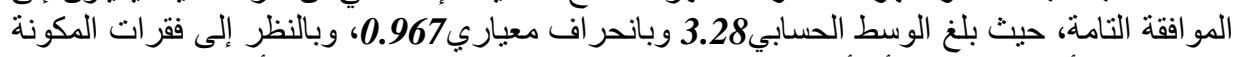

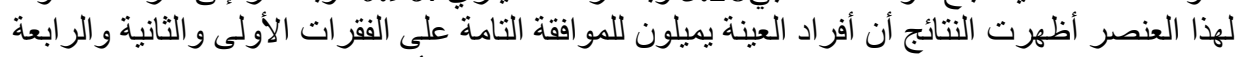

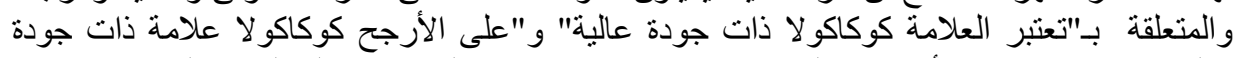

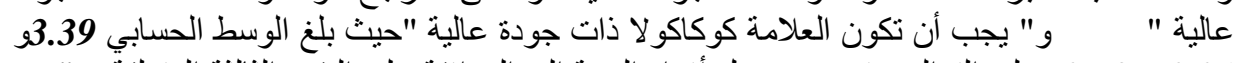

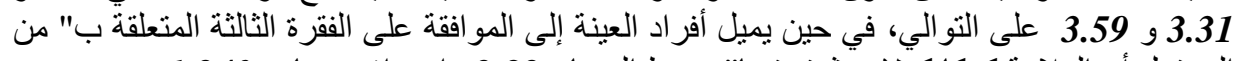

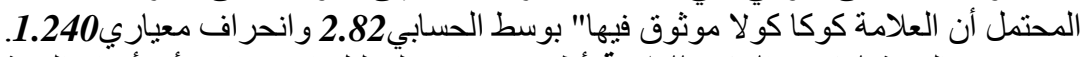

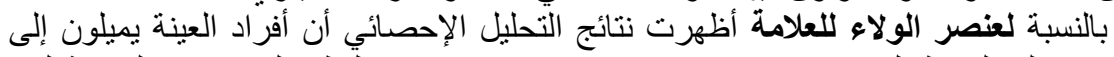

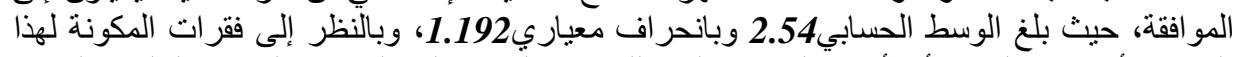

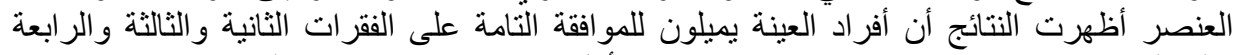

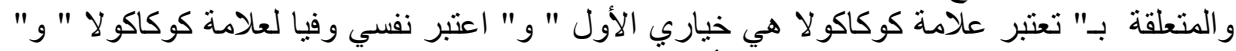

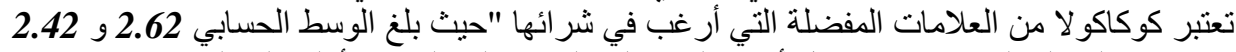

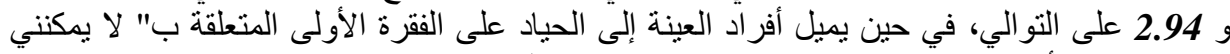

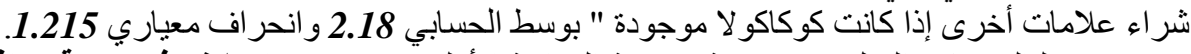

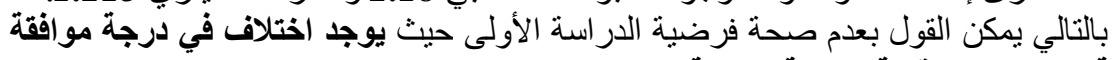

أفر اد العينة على عناصر قيمة العلامة للعلامة كوكاكولا.

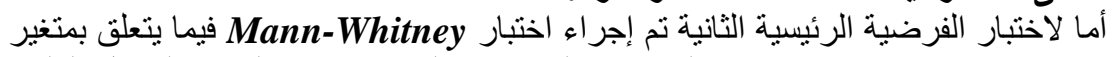

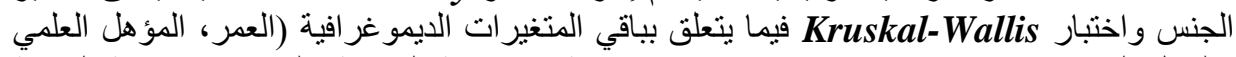
و الدخل الثهري) حيث اتضح عدم وجود فروق معنوية في درجة الموافقة على عنى عناصر قيمة العلامةً 
اللعلامة كوكاكو لا بالنسبة للجنس ذللك أن قيمة (Z=-0.329) -

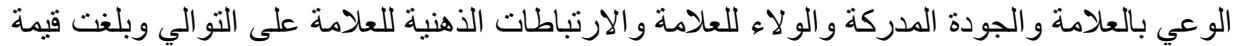

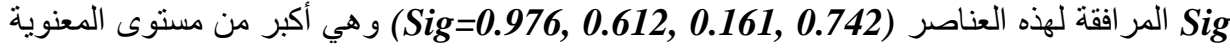
0.05

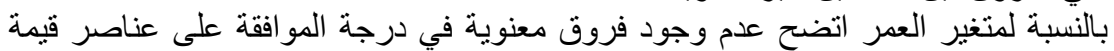

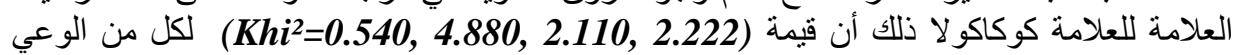

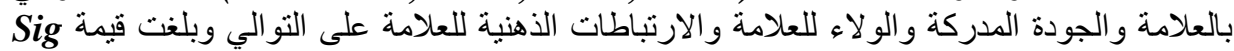
المر افقة لهذه العناصر (Sig=0.528, 0.910, 0. 181, 0.550) و هي أكبر من مستوى المعنوية 0.05 وبالتالي الفروق بين الفئات العمرية غير معنوية.

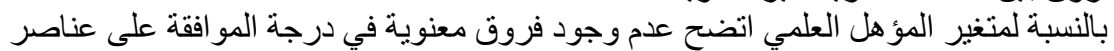

قيمة العلامة للعلامة كوكاكو لا ذلك أن قيمة

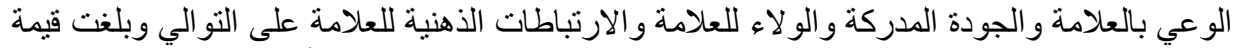

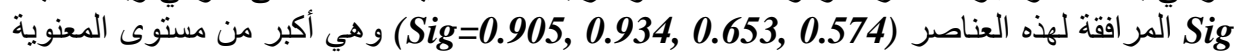
0.05 وبالتالي الفروق بين المؤهلات العلمية غير معنوية.

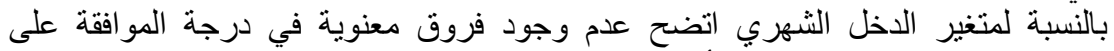

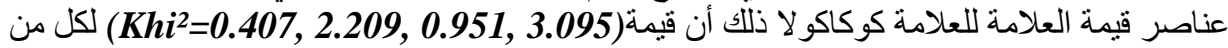

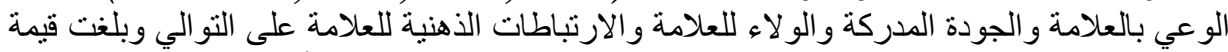

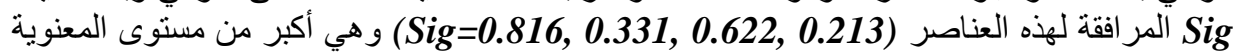
0.05

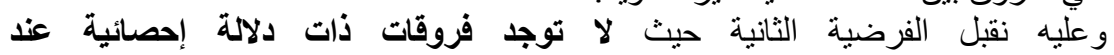

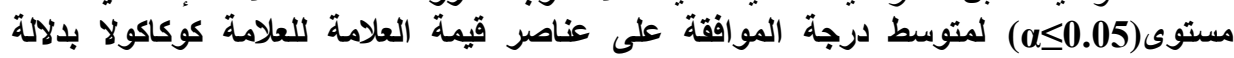

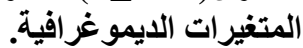
الخاتمة

تتشهد الأسواق الحالية اشتداد المنافسة بين مختلف المؤسسات وفي مختلف القطاعات

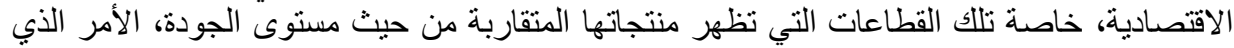

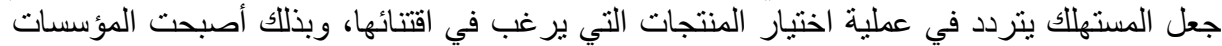

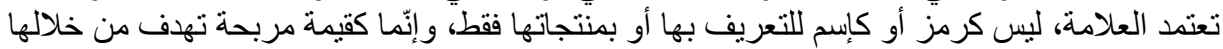
إلى تحسين منتجاتها لتكون الأفضل في نظر زبائنها ما يسهل الحفاظ على و لائهم وكذللك اكتساب ولاء خلاء

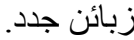

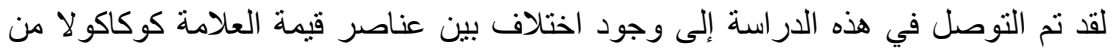

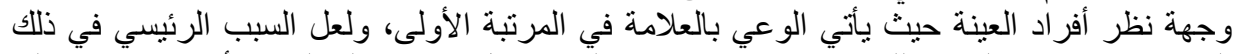

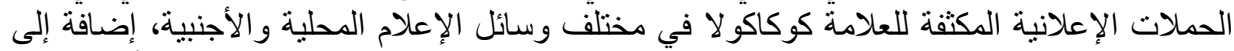

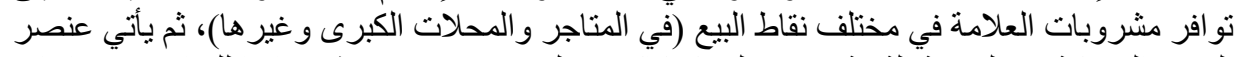

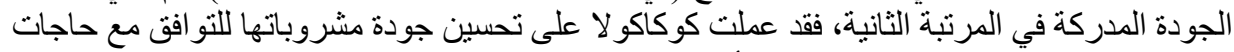

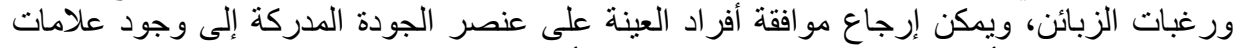

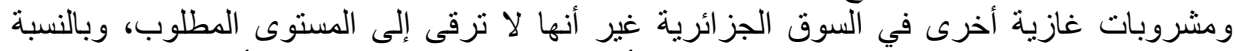

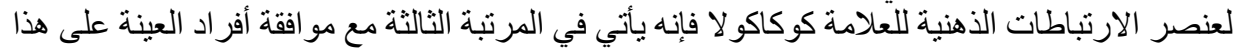

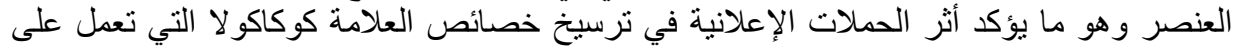

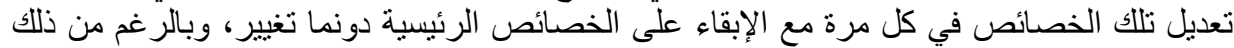

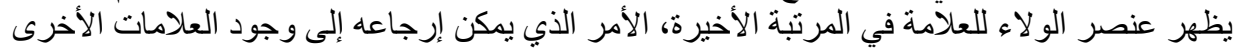

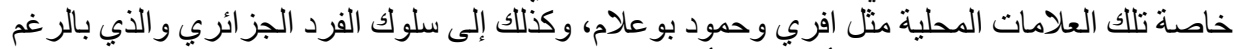

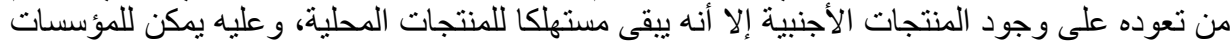


المحلية الناشطة في سوق المشروبات الغازية أن تستفيد من ذلك وأن تعمل على جعل علاماتها أكبر قيمة

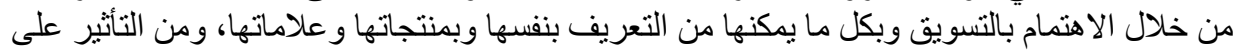

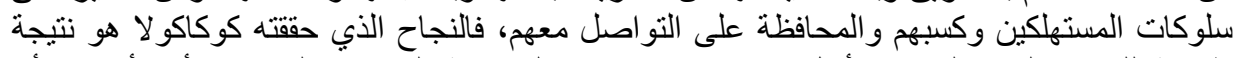

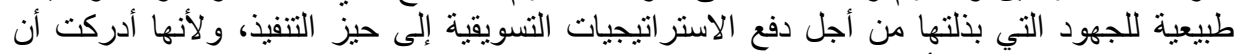
نجاحها يتوقف بالدرجة الأولى على المستهلاك.

(1)-Malaval Philippe, Stratégie et gestion de la marque industrielle, produits et services business to business, Publi-union, Paris, 1998, P1.

(2)-Kotler Philip et al,Marketing management,12 èmédition,Pearson Education,Paris,2006, P314.

(3)-Lewi Georges, Branding management : la marque, de l'idée à l'action, Pearson éducation, Paris, 2005, P12.

(4)-Dioux Jacques and Dupuis Marc, La distribution, Stratégie des groupes et marketing des enseignes, Pearson Education, Paris, 2005, P402.

(5)-Jourdan Philippe, De la marque en capitales vers le capital marque : quoi de neuf depuis les travaux du MSI, P4-5, Site web: http://www.cerag.org/posted/publi/fichier/357/2002-06.pdf.

(6)-Czellar Sandor and Denis Jean- Emille, Un modèle intégrateur du capitalclient de la marque, une perspective psyco-cognitive, P5 Site web:

http://hec.info-unige.ch/recherches_publications/cahiers/2001/2001.08.pdf .

(7)-Czellar Sandor, Capital de marque : concepts, construits et mesures, Site web:

http://market.unige.ch/docs_online/papers_ass_Denis/1997.pdf.

(8)-Yoo Boonghee and Donthu Naveen ,Developing and validating a multidimensional consumer -based brand equity scale ,Journal of business research , 52 ,2001, P 3.

(9)-Korchia Michael, Une nouvelle typologie de l'image de marque, actes du16 ème congres de l'association française du marketing, Montréal, Mai ,2000.

(10)-Korchia Michael, Connaissances des marques stockées en mémoire par les consommateurs :modèle théorique et test empirique, Université de droit ,d'économie et des sciences économiques et de gestion d'Aix Marseille , centre d'études et de recherche sur organisations et la gestion, Institut d'administration des entreprises 2001, P107, Site web : http://www.watoowatoo.net/mkgr/papers/these.pdf .

(11)-Ratier Michel, L'image en marketing: cadre théorique d'un concept multidimensionnel, Centre de recherche en gestion, Cahier de recherche $\mathrm{N}^{\circ} 5$ ,2002-152, IAE de Toulouse, Novembre, 2002, P16-17, Site web:http://www.univ-tlse1.fr/iae/files/154 pdf.pdf .

(12)-Mohammad Reza Jalilvand et al, the effect of brand equity components on purchase intention, an application of Aaker' $s$ model in the automobile 
industry, international business and management, volume 2 ,number $2,2001, \mathrm{P}$ 150.

(13)-Yoo Boonghee and Donthu Naveen ,Developing and validating a multidimensional consumer -based brand equity scale ,Op .cit, P3.

(14)-Lendrevie Jacques and Brochand Bernard, Publicitor,5 éme édition, Dalloz,Paris,2001, P146.

(15)-Dimitriadis Serge, Le management de la marque : vecteur de croissance,

Les éditions d'organisation, Paris, 1994, P34-35.

(16)-Idem.

(17)-Walliser Elisabeth, La mesure comptable des marques, Vuibert, Paris, 2001, P12

(18)- Jourdan Philippe, De la marque en capitales vers le capital marque : quoi de neuf depuis les travaux du MSI, Op.cit, P8.

(19)-Ries Al and Ries Laura, Les 20 lois du capital marque, construire et conserver des marques fortes et durables, Dunod, Paris, 2000, P 78.

(20)-Ratier Michel, L'image de marque à la frontière de nombreux concepts, centre de recherche en gestion, Cahier de recherche $\mathrm{N}^{\circ}$ 2003-158, IAE de

Toulouse, Juillet 2003,P21, Site web : http://www.iae-toulouse

.fr/files/160_pdf.pdf .

(21)-Logié Bernard and Logié -Naville Dora, Leur nom est une marque, Les éditions d'organisation, Paris, 2002, P76.

(22)-Jourdan Philippe, De la marque en capitales vers le capital marque : quoi de neuf depuis les travaux du MSI, Op.cit, P11.

(23)-Temessek Azza, Une approche intégrative pour l'étude des antécédents de la fidélité à la marque, Colloque ATM, 2004, Site web: http://www.mrstdc.gov.tn/association/2colloque_ass_atm/them_session.pdf .

(24)-Laurent François, Les études de marché, comprendre le client .Les éditions d'organisation, Paris, 2001, P192.

$$
\text { الجامعية، الجزائر، عبسى، سلوك المستهلك عوامل التأثير النفسية، الجزء الثناني، ديو ان المطبو عات }
$$

(26)-Malaval Philippe, Stratégie et gestion de la marque industrielle, produits et services business to business, Op.cit, 1998, P123.

(27)-Jourdan Philippe, De la marque en capitales vers le capital marque : quoi de neuf depuis les travaux du MSI, Op.cit, P11.

(28)-Walliser Elisabeth, La mesure comptable des marques, Op.cit, P 12.

(29)-Ministère de la PME et de l'Artisanat Commission \&Européenne, étude de la filière boisson Algérie, Rapport Principal, Euro Développement Pme, Alger 2005, P 15-16, Site web :

http://www.andpme.org.dz/index.php/en/?option=com_docman.

(30)-Ministre de l'industrie, de la petite et moyenne entreprise et de la promotion de l'investissement, Programme d'appui aux PME/PMI et la maitrise des technologies d'information et de communication PME II, La 
filière boissons en Algérie 2012, Période de l'étude : Mars-juillet 2012 PMEII, Alger - Juin 2013 ,P111

(31)-Ibid, P8.

* Hespérides acidulées ou moyen acides citrique, tartrique ou lactique.

(•) GBA : Groupe Boissons d'Algérie

ENAJUC : Entreprise Nationale des Jus et Concentrés.

ONCV :Office National de Commercialisation des Vins

(32)-بن تفات عبد الحق، دور التسويق الزراعي في تحسين الأمن الغذائي مع الإشارة إلى حالة

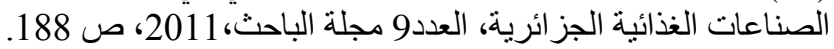

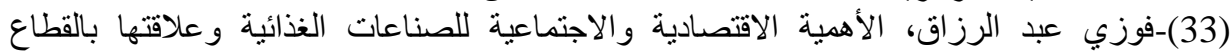

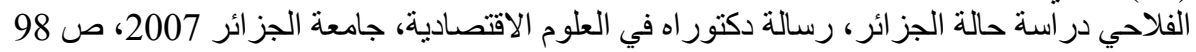

(•) BGI : Brasseries et Glacières Internationales

(34)-Ministre de l'industrie, de la petite et moyenne entreprise et de la promotion de l'investissement, Programme d'appui aux PME/PMI et la maitrise des technologies d'information et de communication PME II, La filière boissons en Algérie 2012, Op ;cit, P82.

${ }^{\bullet}$ SGP COJUB : Les Sociétés de Gestion des Participations (Conserveries, Jus et Boissons)

(35)-Samira Imadalou, Algérie : Privatisation dans le secteur de l'agroalimentaire Cevital et Flash acquièrent la conserverie d'El Kseur et la brasserie d'El Harrach, all Africa - 21 août 2006, Site web :http://houblon.net

${ }^{(\bullet)}$ APAB : L'Association des Producteurs Algériens de Boissons (36)-Ministère de la PME et de l'Artisanat et Commission Européenne, étude de la filière boisson Algérie 2005, Op.cit, P21.

(37)-Ministre de l'industrie, de la petite et moyenne entreprise et de la promotion de l'investissement, Programme d'appui aux PME/PMI et la maitrise des technologies d'information et de communication PME II, Op.cit, P30.

(38)-Panorama sectoriel à l'international, Filière boissons gazeuses et jus de fruits information des entreprise sur les marchés extérieures, Alger /Optime port, juillet 2010, Site web

http://ubifrance.typepad.fr/files/invit_pano_sect_bois_-jus7juil3.pdf.

(39)-Premiers résultats de l'Enquête Nationale sur les Dépenses de Consommation et le Niveau de Vie des Ménages 2011, Office National des statistiques, Alger, 2011, P2, Site web :

www.ons.dz/IMG/pdf/Donnee_statistique_Avril_2013_TER3.pdf.

(40)-جمال محد شاكر، التحليل الإحصائي للبيانات باستخدامSPSS ، كPS. الطبعة الأولى، الدار الجامعية، 